\title{
Spectrum Handoff Method Based on Extenics for Cognitive Radio Sensor Networks
}

\author{
http://dx.doi.org/10.3991/ijoe.v12i03.5418 \\ Yonghua Wang ${ }^{1}$, Pin Wan ${ }^{2,3}$, Sheng Ouyang ${ }^{2}$,Fei Yuan ${ }^{4}$ and Yuli Fu ${ }^{1,5}$ \\ ${ }^{1}$ South China University of Technology, Guangzhou, China \\ ${ }^{2}$ Guangdong University of Technology, Guangzhou, China \\ ${ }^{3}$ National Engineering Research Center for Mobile Communication, Guangzhou, China \\ ${ }^{4}$ Guangdong Polytechnic Normal University, Guangzhou, China \\ ${ }^{5}$ South-Central University for Nationalities, Wuhan, China
}

\begin{abstract}
A spectrum handoff model and optimal channel decision method based on Extenics have been proposed in order to resolve the optimal channel decision problem of spectrum handoff in Cognitive Radio Sensor Networks. The method of matter-element Extenics is used to analyses the spectrum handoff process. The channel state through the spectrum sensing and status information of the primary users is analyzed and transformed by matter-element Extenics. Then the parameters to determine the best channel are calculated on the basis of Extenics correlation function. Finally the optimal channel is selected. The simulation results show that the proposed method can improve spectrum utilization efficiency of Cognitive Radio Sensor Networks and can reduce the interrupt probability of second users.
\end{abstract}

Index Terms-Cognitive Radio Sensor Networks, Extenics, Spectrum Handoff, Decision

\section{INTRODUCTION}

Cognitive Radio Sensor Networks (CRSN) are composed of Wireless Sensor Networks (WSN) and Cognitive Radio(CR) technology, can effectively solve the problem that the available spectrum resources are limited in WSN $[1,2]$. CRSN have the following characteristics [3]: low energy consumption, simple hardware, self-organization, event-driven, etc. In CRSN, the SUs (second users, SU) can use the spectrum of PUs (Primary users, PU) only in the case of don't have interfere with PUs. Once the PU appears, SU must vacate the occupied frequency band, search and switch to another idle channel, this process is called spectrum handoff [4]. There are three basic spectrum handoff models $[5,6]$ : stay and wait, predetermined target channel list, sensing idle spectrums instantly.

In spectrum handoff, the most important problem is how to select the best candidate channel. The spectrum handoff is a multi-attribute comprehensive decision, need to consider various parameters to make the judgment such as the characteristics of channel, the accessing probability of PUs, the requirements of SUs and communication quality and so on [7]. And the spectrum information sensed by the CRSN nodes has significant uncertainty. It thus leads to the parameters have some uncertainty. Existing decision methods including fuzzy method[4,8], the simple weighted algorithm[9], the dynamic weight decision method[10], analytic hierarchy process, the technique for order preference by similarity to ideal solution [11] etc. However, the above methods require more parameters and the computation is relatively complexity. Because the
CRSN have the characteristics such as simple hardware and low computational ability, and the Extenics is suitable for multi-attribute judgment under uncertainty and low computation required $[12,13]$, this paper proposed a spectrum handoff model and an optimal channel decision method based on Extenics comprehensive considering various factors of spectrum handoff.

\section{SYSTEM MODEL}

Extenics is a science using a formal model to research the extension possibility of things, the rules and methods of innovation, and applying all of them to solve contradictory problems. It overcomes the limitation of the classical set and fuzzy set and has developed from only can describe the deterministic things to can describe the things in a state of flux [12]. Therefore it is suitable for dealing with uncertainty information. Extenics employ the correlation function to depict the degree of some properties that the element in the domain has. And it can quantitative and objective describes the degrees of some characteristics of the elements and the quantitative change and qualitative change.

This paper proposed an optimal channel judgment model based on Extenics for CRSN. After the spectrum sensing to PUs, SUs predict the channel state and choose the optimal channel in the period. Within each cycle, SUs will make an anticipation to judge the possibility that the PUs access to the candidate channel. Thus, SUs can switch to another idle channel in advance. This would reduce the colliding probability with PUs and the switching frequency. Considering the actual needs, we proposed a spectrum handoff model, as shown in Figure 1.

\section{The Best Access ChANNEL JUdGMENT BASED ON EXTENICS}

\section{A. The description of Extenics matter-element}

As the spectrum access, the known parameters are the duration of the SUs needs to transfer information, the communication regular pattern of PUs and the channel state information of PUs obtained by spectrum sensing. The desired result is to choose the most appropriate channel among the candidate channels in order to best meet the communication needs of SU. The parameters described by matter-element are as follows:

The matter-element of known conditions are: 
PAPER

SPECTRUM HANDOFF METHOd BASED ON EXTENICS FOR COGNITIVE RADIO SENSOR NeTwORKS

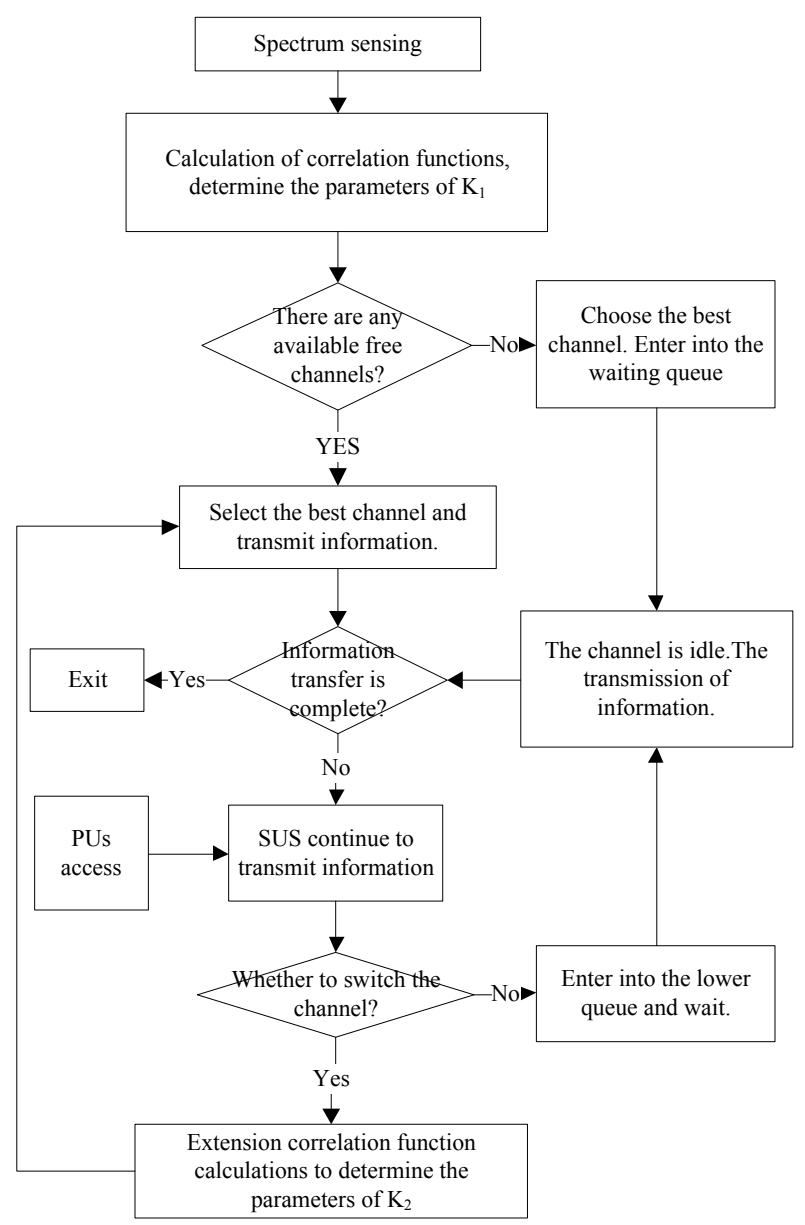

Figure 1. The spectrum handoff model based on Extenics

$R_{1}=(S U S$ The communication time $X)$

$R_{2}=\left(\begin{array}{ccc}P U s & \text { Channel condition } & H \\ & \text { Communication regular pattern } & F\end{array}\right)$

The objective is to select the best channel for cognitive users. So the matter-element of the objective is:

\section{$O=($ Channel The optimal channel $i)$}

The condition matter-element is the description of SUs and PUs. It couldn't directly judge which is the optimal channel for SUs. Since we couldn't directly calculate the objective matter-element from the condition matterelement, thus the extension transformation is needed to make them into a more direct calculation.

\section{B. The transformation of extension matter-element}

SUs can judge whether the channel exists PUs via spectrum sensing, that is $H_{0}$ (PUs don't access the channel, the channel is idle) or $H_{1}$ (PUs access the channel, the channel is busy). When the channel is idle, PUs may access the channel again. Then the idle channel will switch to the busy status, thus there exists the duration of idle. When the channel is busy, PUs complete the communica- tion after a period time, and the channel will switch to the idle from busy, thus there exists the duration of busy.

Therefore, PUs matter-element $R_{2}$ can be expanded the through extension divergence:

$$
\begin{aligned}
& R_{2}=\left(\begin{array}{ccc}
P U S & \text { Channel condition } & H \\
& \text { Communication regular pattern } & F
\end{array}\right) \\
& \Rightarrow\left(\begin{array}{ccc}
P U S & \text { Channel condition } & H \\
& \text { The busy time } & Z \\
& \text { The idle time } & Y
\end{array}\right)
\end{aligned}
$$

According to [6], the communication time of SUs and PUs obeys exponential distribution. Now we can know the range of the SUs and PUs' communication time (the duration of busy). But we don't know the range of the idle time. Thus via the equation (4), through the matterelement's divergent, $R_{2}$ can be transformed to:

$$
\begin{aligned}
& R_{2}=\left(\begin{array}{cc}
\text { PUs } & \begin{array}{c}
\text { Channel condition } \\
\text { The busy time }
\end{array} \\
& Z \\
\text { The idle time } & Y
\end{array}\right) \\
& \Rightarrow\left(\begin{array}{ccc}
\text { Channel } i & \text { Channel condition } & H \\
& \text { The busy time } & Z_{i} \\
& \text { The idle probability } & p_{i}
\end{array}\right) \\
& \Rightarrow\left(\begin{array}{ccc}
\text { Channel } i & \text { Channel condition } & H \\
& \text { The busy time } & Z_{i} \\
& \text { PUs access probability } & \gamma_{i}
\end{array}\right)
\end{aligned}
$$

According equation (1) and (5), the condition matterelement is:

$R_{2}=\left(\begin{array}{ccc}\text { Channel } i & \text { Channel condition } & H \\ & \text { The busy time } & Z_{i} \\ & \text { PUs access probability } & \gamma_{i}\end{array}\right)$

The objective matter-element is:

$$
O=(\text { Channel The optimal channel } i)
$$

Among $i=1,2,3, \mathrm{~L} N, N$ is the number of candidate channels.

\section{Calculation of the correlation function}

Since communication time obeys exponential distribution, the cumulative distribution function of exponential distribution is:

$$
F(x ; \eta)=1-e^{-\eta x}, x \geq 0
$$

In order to make $X$ greater than $99.9 \%$, then $F(x ; \eta)=1-e^{-\eta x}=0.999$, we can obtain $x=\frac{3 \ln 10}{\eta}$. 
Therefore, the number range of $X$ is $x \in\left[0, \frac{3 \ln 10}{\eta}\right]$, the expectation value is $E(X)=1 / \eta$, where $\eta$ is the exponential distribution parameter of communication time $X$.

Similarly, PUs' communication time obey exponential distribution. The PUs' communication time on the candidate channel is $Z \in\left[0, \frac{3 \ln 10}{\mu}\right]$, and its expectation value is $E(Z)=1 / \mu$. Where $\mu$ is the exponential distribution parameter of PUs.

The probability of PUs access to the channel is between 0 and 1 , according (1) and (6):

$R_{1}=\left(S U S\right.$ The communication time $\left.\left[0, \frac{3 \ln 10}{\eta}\right]\right)$

$$
R_{2}=\left(\begin{array}{ccc}
\text { Channel } i & \text { Channel condition } & 0 / 1 \\
& \text { The busy time } Z_{i} & {\left[0, \frac{3 \ln 10}{\mu}\right]} \\
& P U \text { s access probability } \gamma_{p}^{i} & {[0,1]}
\end{array}\right)(10)
$$

According to the channel selection model, the objective matter-element aim to select the optimal channel, according (6), we can transform target matter-element to:

$O=\left(\begin{array}{ccc}\text { The optimal channel } & \text { State } & 0 / 1 \\ & \text { PUs access } & \\ & \text { probability } & \gamma_{0} \\ & P U \text { s comm. time } & \mathrm{z}_{0}\end{array}\right)$ (11)

Since the optimal channel needs to be selected. And for candidate channels, the optimal channel means the channel is free, the lowest probability of PUs accessed, the shortest time that PUs communicated. According to (11), the optimal channel is:

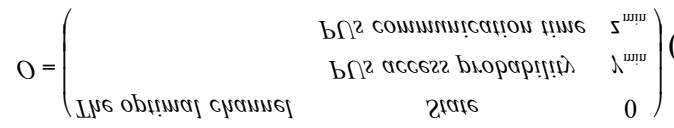

These three parameters of each candidate channel are calculated on the basis of the correlation function. Then the optimal channel can be selected.

For channel's state, idle is well, so:

$$
\rho_{i}^{H}=1-H
$$

When the channel is busy, then $H=1$. When the channel is idle, $H=0$.

For the probability that the PUs accessed, the lower the better, so:

$$
\rho_{i}^{\lambda}=\frac{\gamma_{\max }-\gamma_{i}}{\gamma_{\max }-\gamma_{\min }}
$$

$\gamma_{\max }$ and $\gamma_{\text {min }}$ express the max and min value respectively in $\left(\gamma_{1}, \gamma_{2}, \gamma_{3}, \mathrm{~L}, \gamma_{N}\right)$. Where $i=1,2,3, \mathrm{~L}, N, N$ is the total number of candidate channels.

As SUs can continue to communicate only after the PUs finished communication, so the shorter the PUs' communication time is the better. Therefore:

$$
\rho_{i}^{z}=\frac{z_{\max }-z_{i}}{z_{\max }-z_{\min }}
$$

In which $z_{\max }$ and $z_{\text {min }}$ express the max and min value respectively in $z_{1}, z_{2}, z_{3}, \mathrm{~L}, z_{N}$.

To obtain the best channel at this time, it has the following discriminant:

$$
K_{1}(i)=\frac{1}{3} \rho_{i}^{H}+\frac{1}{3} \rho_{i}^{\lambda}+\frac{1}{3} \rho_{i}^{z}
$$

By synthesizing (13), (14), (15) and (16), the determining parameters $K_{1}(i)$ can be calculated. Select the channel corresponding to the max value in $K_{1}(i)$, then the channel is the optimal channel.

\section{The Optimal Channel JUdGMENT When SPECTRUM HAND-OFF}

When the SU in the process of transmission, it may encounter a re-access of the PUs. At the time, there are two choices. The first is that no switching. SU will wait in the lower queue. The second is that calculation the correlation function based on the parameters, and chooses the optimal channel. Whether to switch is based on the calculation. If the current channel in all candidate channels is the best, it doesn't need to switch. Otherwise switch.

When SUs access a channel, to avoid colliding with PUs once the PUs access the channel again, a predictive analysis on the channel is needed. And in order to achieve the detection results, the forecast period is supposed to be $\Delta t$. The parameters in the calculation need considered as follows: the accessing probability of PUs and the communication time after PUs access the channel. Then according to the parameters of each channel, the correlation of each parameter is calculated based on the correlation function. The final judgment result is obtained according to the weight of each parameter, and then the optimal channel is derived.

The channel initial state as Equation (6) described, is known conditions. The value need to calculate is the channel state after $\Delta t$ time. So the matter-element of channel is:

$$
R_{4}=\left(\begin{array}{lll}
\text { Channel } & \text { The probability of Channel is } H_{1} & P_{H_{1}} \\
& \text { The probability of Channel is } H_{0} & P_{H_{0}}
\end{array}\right) \text { (17) }
$$

The time length occupied by SUs is $\mathrm{x}$. The forecast period is supposed to be $\Delta t$. When $\Delta t$ is smaller, the number of channel prediction is more, and the accuracy of detection results is higher. When $\Delta t$ is too large, it will result fail to estimate the accessing probability of PU in the forecast period and increase the probability of collision. Therefore, we need to 
choose the appropriate forecast period $\Delta t$ after many experiments.

After every $\Delta t$, the state probability of channel will change. So it will need judge whether to switch channel after every $\Delta t$.To prevent excessive switch, we need to consider the channel's current state when selecting channels. If after $\Delta t$ the channel is still idle, SUs can continue to communicate on the channel. If after $\Delta t$ the channel is busy, SUs should select the optimal idle channel from the remaining idle channel.

When $H=0$, after $\Delta t$ the probability of the channel is still idle is:

$$
p\left(H_{00}\right)=\frac{(1-\gamma) z-\gamma \Delta t}{z}
$$

The probability of the channel turns to busy:

$$
p\left(H_{01}\right)=\frac{\gamma^{2} \Delta t}{(1-\gamma) z}
$$

When $H=1$, after $\Delta t$ the probability of the channel is still busy is:

$$
p\left(H_{11}\right)=\frac{\gamma(z-\Delta t)}{z}
$$

The probability of the channel turns to idle is:

$$
p\left(H_{10}\right)=\frac{(1-\gamma) \Delta t}{z}
$$

When $H=H_{0}$, the probability of the channel is idle is less than or equal to 0 , then $H=H_{1}$.

When $H=H_{1}$, the probability of the channel is busy is less than or equal to $0, H=H_{0}$.

According to (17), we can get the channel matterelement:

$$
R_{4}=\left(\begin{array}{ccc}
\text { Channel } & H_{0} \rightarrow \text { Channel idle probability } & p\left(H_{00}\right) \\
& H_{0} \rightarrow \text { Channel busy probability } & p\left(H_{01}\right) \\
H_{1} & \rightarrow \text { Channel idle probability } & p\left(H_{10}\right) \\
& H_{1} \rightarrow \text { Channel busy probability } & p\left(H_{11}\right)
\end{array}\right)(22)
$$

According to the (6) and (22), the condition matterelement can be transform to the follows formula:

$$
\begin{aligned}
& \text { PUs communication time } \\
& \text { PUs access probability } \\
& H_{0} \rightarrow \text { Channel idle probability } \\
& H_{0} \rightarrow \text { Channel busy probability } \\
& H_{1} \rightarrow \text { Channel idle probability } \\
& H_{1} \rightarrow \text { Channel busy probability } \\
& \left.\begin{array}{c}
y_{i} \\
\gamma_{i} \\
p\left(H_{00}\right) \\
p\left(H_{01}\right) \\
p\left(H_{10}\right) \\
p\left(H_{11}\right)
\end{array}\right)
\end{aligned}
$$

The parameters of each channel are different. When the idle probability is higher and the busy probability is lower, the probability that the channel is selected will be higher. $p_{\max 0}$ and $p_{\min 0}$ express max and min idle probability of the channel in the candidate channels respectively; $p_{\max 1}$ and $p_{\min 1}$ express max and min busy probability respectively. Therefor according to (7), the optimal channel matterelement is:

$$
O=\left(\begin{array}{ccc}
\text { Channel } i & \text { Channel idle probability } & p_{\max 0} \\
& \text { Channel busy probability } & p_{\min 1}
\end{array}\right)
$$

Suppose $p_{i 0}$ is the idle probability of channel $i, p_{i 1}$ is the busy probability of channel $i$.Therefor the correlation of idle channel is:

$$
\rho\left(H_{0}\right)=\frac{p_{i 0}-p_{\min 0}}{p_{\max 0}-p_{\min 0}}
$$

The correlation of busy channel is:

$$
\rho\left(H_{1}\right)=\frac{p_{\max 1}-p_{i 1}}{p_{\max 1}-p_{\min 1}}
$$

After every $\Delta t$, the channel is selected according the channel preferred degree:

$$
K_{2}=\frac{1}{2} \rho\left(H_{0}\right)+\frac{1}{2} \rho\left(H_{1}\right)
$$

According to (25), (26) and (27), the parameter of judgment can be calculated, since every candidate channel's is different, the optimal channel is the channel that has the largest value in the candidate.

\section{SIMULATION ANALYSES}

Monte Carlo simulations are carried out for the proposed method with Matlab software. Suppose that the simulation number is 2000 and the number of the candidate channel $N=5$. The communication time of PUs and SUs obey to the exponent distribution. The accessing probability of PUs obeys to Poisson distribution, and the scope of that probability is $\gamma \in(0,1)$. In the following simulations, the performances of channel choice under the different accessed probability for PUs are analyzed.

Figure 2 represent under different accessing probability of PUs, the changes of the interrupt probability of SUs based on the Extenics method and random selection method. The communication time of SUs obey $\eta=0.05$ exponent distribution. The communication time of PUs obey $\mu=0.09$ exponent distribution. The forecast period $\Delta t=0.5$. The initial state of candidate channel is supposed to be $(0,0,1,1,0)$, where 0 represents idle and 1 represent busy. According to the analysis of spectrum handoff, the probability of SUs be interrupted is the lower the better. Figure 2 shows that the performance of the method on the Extenics is better than the random selection method. The interrupted probability of SU is increased as PUs' accessing probability increased. Therefore the SU should choose the channel that has the low accessing probability. 
Figure 3 is the spectrum handoff probability of SUs under the different accessing probability of PUs. PUs access the channel again will affect the SUs when the SUs select the optimal channel to communication, therefore the quitting and switching probability of the SUs will increase when the accessing probability of PUs increased. In simulation, the performances of the cycle prediction method based on Extenics and the passive selection method are compared. The method based on Extenics has lower switching probability under the equal condition. This method not only prevents the signal delay caused by repeatedly switching but also save the energy consumption.

When SUs are in the process of signal transmission, if PUs access the channel. SUs should switch to the free channel or waiting in the low level queue until the PUs finish communication. This will increase the communication time of SUs. In order to ensure the quality of the communication, SUs should finish the communication in the shortest time. In order to make full use of the idle channel we should improve the spectrum utilization. The probability of spectrum utilization for SUs is decreased as the accessing probability of PUs increased. Figure 4 shows the spectrum utilization of SUs under the different accessing probability of PUs. We can see that the method based on the Extenics has the higher probability of spectrum utilization than other methods.

Figure 5 shows the spectrum utilization of SUs under the condition that the communication time of SUs are

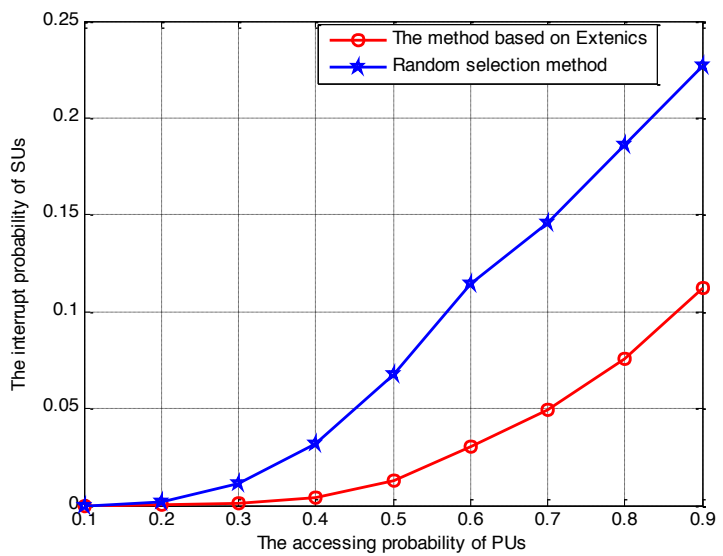

Figure 2. The interrupt probability of SUs under different accessing probability of PUs

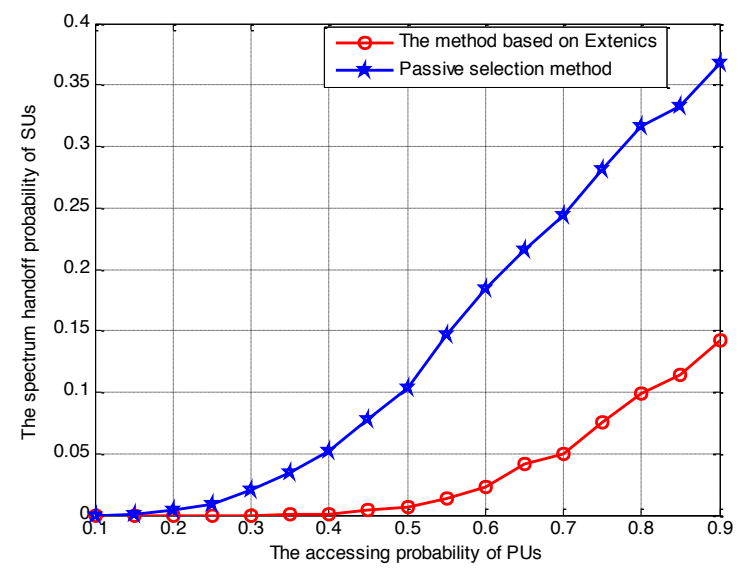

Figure 3. The spectrum handoff probability of SUs under the different accessing probability of PUs fixed and communication time parameter of PUs is changed. Supposed the PUs' communication time parameter is $\mu=0.03, \mu=0.06, \mu=0.09$ and $\mu=0.12$ respectively. From the Figure 5 we can see that the spectrum utilization increases as the parameter $\mu$ increases. In the whole channel choice process, the PUs' communication time will decrease when the $\mu$ increased. When SUs are waiting for the PUs communication, the length of waiting time is actually decreased. According to the binary hypothesis of channels, when the communication time of the PUs shortened, the length of channel idle time actually increased. Therefore the spectrum utilization for SUs will increase, which is consistent with the actual result.

\section{CONCLUSIONS}

This paper proposed a period prediction spectrum handoff scheme based on the Extenics and an optimal channel selection methods for CRSN. Using the Extenics matter-element method to analyse the spectrum handoff process. The channel state information sensed by SUs and the spectrum information of PUs is carried out by Extenics analysis and matter element transformation. Then the optimal channel parameters are calculated on the basis of the Extenics correlation function. Finally, the optimal channel was selected. The simulation results show that this scheme can improve the probability of spectrum utilization and decrease the interruption probability of SUs.

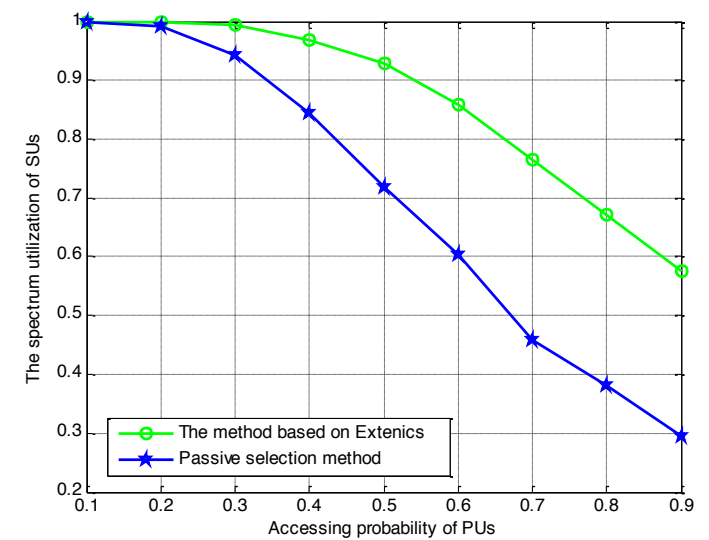

Figure 4. The spectrum utilization of SUs under the different accessing probability of PUs

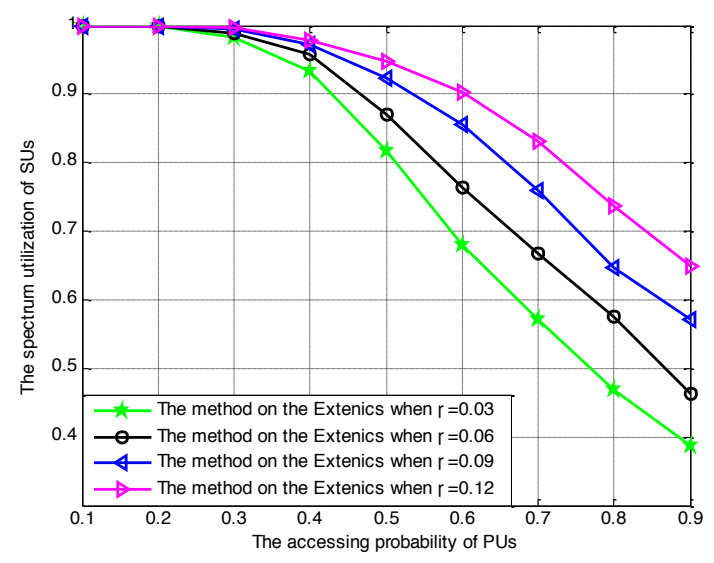

Figure 5. Spectrum utilization of SUs under $\mu=0.03, \mu=0.06$,

$$
\mu=0.09 \text { and } \mu=0.12
$$


PAPER

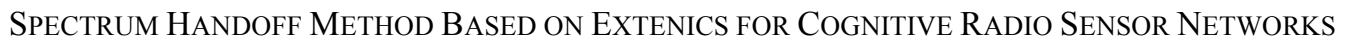

\section{REFERENCES}

[1] O.B. AKAN, O. KARLI, and O. ERGUL, "Cognitive Radio Sensor Networks," IEEE Networks, no.7-8,pp.34-40,2009.

[2] Y Wang,J Yang,L Cheng,P Wan, and B Zhang, "Survey on Wireless Cognitive Radio Sensor Networks ," Computer Science, vol.38, no.7,pp. 41-45,2011.

[3] H Zhang,Z Zhao,H Dai,R Yin, and X Chen, "Distributed Spectrum-Aware Clustering in Cognitive Radio Sensor Networks," IEEE GLOBECOM, Houston, TX, USA, pp.5-9 ,Dec. 1-6,2011.

[4] E.Z.Tragos,S.Zeadally, A.G.Fragkiadakis, V.A. Siris, "Spectrum Assignment in Cognitive Radio Networks: A Comprehensive Survey," IEEE Communications Surveys \& Tutorials, vol.15, no.3,pp.1108-1135,2013. http://dx.doi.org/10.1109/SURV.2012.12 1112.00047

[5] L Wang, C.ANDERSON, "On the performance of spectrum handoff for link maintenance in cognitive radio," 3rd International Symposium on Wireless Pervasive Computing, 2008. Santorini pp.670-674, May 2008.

[6] X Li, (REZA) ZEKAVAT, A.SEYED, "Traffic Pattern Prediction and Performance Investigation for Cognitive Radio Systems," 2008 IEEE Wireless Communications and Networking Conference(WCNC 2008), Las Vegas, NV,pp.894-899, March 31 2008April 3 2008. http://dx.doi.org/10.1109/WCNC.2008.163

[7] S YI, X JIANG, "ProSpect: A Proactive Spectrum Handoff Framework for Cognitive Radio Ad Hoc Networks without Common Control Channel," IEEE Transactions on Mobile Computing, vol.11, no.7, pp.1127-1139,2012. http://dx.doi.org/10.1109/TMC. $\underline{2011.140}$

[8] X Fu,Y Zhou, "A Handoff Decision Algorithm Based on Fuzzy Neural Control in CRN," Wireless Communication Technology,no.1,pp.10-13,2013.

[9] Q Wu,Y Xian,C Xu, and H Qin, "Study on handover channel selection in cognitive radio based on multiattribute making decision," Computer Engineering and Applications, vol.47, no.27, pp.158-160,2011.
[10] Z Zhao, L Zhang, "Spectrum Handoff Based on Dynamic Weights Adjustment in Cognitive Radio Network,"Journal of Signal Processing,vol.30,no.3,pp.276-280,2014.

[11] J Jiang,K Wang,X Fu, and Z Xiong, "A Novel Spectrum Handoff Scheme Based on TOPSIS Channel Order Algorithm," Telecommunication Engineering,vol.52,no.5,pp.761-765,2012.

[12] W Cai, C Yang, "Basic theory and methodology on Extenics. Chin Sci Bull," vol.58, pp.1190-1199,2013. http://dx.doi.org/10.1360/ 972012-1472

[13] C Yang, W Cai, Extenics: Theory, Method and Application. Columbus: Educational Publisher, 2013

\section{AUTHORS}

Yonghua Wang is with School of Electronic and Information Engineering, South China University of Technology, Guangzhou 510641, China (e-mail: sjzwyh@163.com).

Pin Wan is with School of Automation, Guangdong University of Technology, Guangzhou 510006, China (email: pinwan2@163.com).

Sheng Ouyang is with School of Automation, Guangdong University of Technology, Guangzhou 510006, China (e-mail: 554849940@qq.com).

Fei Yuan is with School of Automation, Guangdong Polytechnic Normal University, Guangzhou 510665, China (e-mail: eric_f_y@foxmail.com)

Yuli Fu is School of Electronic and Information Engineering, South China University of Technology, Guangzhou 510641, China (e-mail: fuyuli@scut.edu.cn).

This work was supported in part by The National Natural Science Foundation of China. (No.61471174), the China Postdoctoral Science Foundation (No.2014M552529, No.2015T80920) and the Youth Foundation of Guangdong University of Technology (No.13QNZD006). Submitted 29 December 2015. Published as resubmitted by the authors 13 February 2016. 